Article Citation Format Ikuomola, A. J (2019): Toward a Secured Multi-Step Permission Based Delegation Model with Chinese Wall Security Policy Journal of Digital Innovations \& Contemp. Res. In Sc., Eng. \& Tech. Vol. 7, No. 1. Pp 11-22

$\begin{array}{r}\text { Article Progress Time Stamps } \\ \text { Article Type: Research Article } \\ \text { Manuscript Received: } 17^{\text {th }} \text { Dec. } 2018 \\ \text { Review Type: Blind } \\ \text { Final Acceptance: } 24^{\text {th }} \text { Dec., } 2018 \\ \text { DOI: dx.doi.org/10.22624/AIMS/DIGITAL/V7N1P2 } \\ \hline\end{array}$

\title{
Toward a Secured Multi-Step Permission Based Delegation Model with Chinese Wall Security Policy
}

\author{
Aderonke J. Ikuomola \\ Department of Mathematical Sciences, \\ Ondo State University of Science and Technology \\ Okitipupa, Nigeria \\ E-mail: deronikng@yahoo.com, aj.ikuomola@osustech.edu.ng
}

\begin{abstract}
The Chinese Wall Security Policy (CWSP) model is one of the access control models, which aims to prevent sensitive information concerning a company being disclosed to competitor companies through the work of financial consultants. PBDM is a flexible delegation model that supports multi-step delegation and revocation in role and permission level. This model uses the can-delegate relation with prerequisite condition to restrict delegates and adds new types of roles and permissions to address permission level delegation requirement. A multi-step or multiple permission based delegation with Chinese wall security policy is a kind of delegation that satisfies Chinese wall security policy (CWSP) as a delegation constraint. This work discusses on how multiple permissions can be delegated with the restriction of CWSP. Firstly, a secure model and algorithm was defined for multiple delegation and revocation of permissions based on the strong CWSP constraint. Secondly, different types of revocation were discussed and finally implementation was done by developing a web based application using PHP that can be used in a commercial world such as an investment or financial house where there exist a conflict of interest and where delegations of permissions in multiple steps are necessary.
\end{abstract}

Keywords: Delegation, Revocation, Permission, Security Policy, Access control

\section{INTRODUCTION}

Quan et al. [11] describes Delegation as a mechanism for assigning privileges as well as other attributes to users. Delegation means that a user (delegator) can give her permissions to other person (delegatee). There are three types of situations in which delegation can take place: backup of roles, decentralization of authority and collaboration of work. Human-to human delegation has received considerable attention recently [19, 4, 20]. The user who performs a delegation is referred to as a "delegator" and the user who receives a delegation is referred to as a "delegatee". PBDM is a flexible delegation model that supports multi-step delegation and revocation in role and permission level. 
A multi-step delegation is performed with the involvement of one or many intermediate parties which can forward the delegation assertion from the delegator to the delegate. Revocation is an important aspect in delegation models. Revocation is of two types: user and automatic revocation. User revocation means revocation can only be performed by delegator or system administrator manually, while automatic revocation means revocation is triggered and performed by system automatically. User revocation can be found in most delegation model, but few support automatic revocation.

[12] was of the opinion that an important requirement of any information management system is to protect data and resources against unauthorized disclosure (secrecy) and unauthorized or improper modifications (integrity), while at the same time ensuring their availability to legitimate users (no denials-of-service). The Chinese Wall policy arises in the financial segment of the commercial sector, which provides consulting services to other companies [13]. The Chinese wall security policy model (CWSP Model) introduced by [1] is an access control model which was stimulated by business operations where client database need to be kept separate from each other. It models the constraints of a firm of professionals such as computer consultants, advertising agents or investment bankers etc. whose partners need to avoid situations where conflicts of interest or insider dealing might become possible [5].

It has been widely applied in commercial world where there exists conflict of interest among different companies. The objective of the Chinese Wall policy is to prevent information flows which cause conflict of interest for individual consultants [6]. Consultants naturally have to deal with confidential company information for their clients. A single consultant should not have access to information about two banks or information about two oil companies, etc., because such insider information creates conflict of interest in the consultant's analysis and disservice to the clients. Insider information about companies of the same type also presents the potential for consultants to abuse such knowledge for personal profit [13].

There are two types of CWSPs: weak and strong CWSP: Weak CWSP can be seen as a normal delegation constraint. This means a user can keep two conflict roles at the same time but she can only activate one role and lost the right of activating the other one forever. So, if a delegatee has roles that conflict with delegation role but she has not activated or is activating these roles, delegation will be allowed. Strong CWSP is stricter than weak CWSP. This means if delegatee already has roles that conflict with delegation role, delegation will not be allowed even if those roles has not been activated before. Strong CWSP goes beyond the basic security restrictions of CWSP and is useful in a situation of multi-step delegation [3].

This work aim at avoiding sensitive information concerning a company being disclosed to competitor companies through the work of financial consultants when delegating permissions in $\mathrm{n}$ - number of steps. In a commercial environment [3], delegation occurs frequently, especially when someone is on a business trip. That means a user (delegator) can transfer her permissions to other person (delegatee) and delegatee can perform these transferred permissions just like the delegator herself. It is difficult to delegate permissions coming from different roles especially in a multi-step delegation for strong CWSP. In this work, a secure model for multi-step permission delegation with the Chinese wall security access control policy as a major constraint was developed. In the rest of this paper, section 2 presents the review of related works done on security policy and model, Section 3 describe the technique employed in the design of a web based system for permission based delegation model with Chinese wall access control security policy. Section 4 and 5 describe the implementation and conclusion respectively. 


\section{RELATED WORKS}

[14] approach the issue of integrity in trusted database systems from the perspectives of modification authorization (mandatory integrity authorization and discretionary integrity authorization) and information consistency (integrity rules, recovery management and concurrency controls). With respect to multilevel security, it is modification authorization that is of interest. Mandatory integrity authorization is based on data integrity classification and user clearances. Discretionary integrity authorization is based on a user's need to modify data. [10] present a formal security policy model designed for the secure multilevel relational database system under the Sea-View project. The Sea-View security model comprises a Mandatory Access Control (MAC) model inner layer and a Trusted Computing Base (TCB) model outer layer. Central to the MAC model is a mandatory security policy and the use of a reference monitor for policy enforcement. The TCB model is responsible for Discretionary Access Control (DAC) beyond that of the MAC model. Integrity protection is dictated by the Program Integrity Policy (Biba Strict Integrity Policy variant) and is enforced by the MAC model.

[21] defines a flexible delegation model that supports multi-step delegation and revocation in role and permission level. In PBDM0, a user can delegate all or part of his/her permissions to delegatees. In PBDM1 and PBDM2, the permission flow is managed by a security administrator with delegeatable role (DBR). RDM2000 and RBDM can be seen as special cases of PBDM. In most cases, a delegator cannot delegate all of it permissions to delegatee. Therefore, a low level user cannot be assigned to high level permissions. In some delegation models, delegation is managed by the delegator him/herself. Through a survey of the literature, [2] define a set of data integrity requirements; control of information-flow, data verification, prevention of fraud and error, and autonomous data validation. With these requirements in mind, they present the design of architecture for data integrity control systems. A Policy Specification Language (PSL) facilitates the specification and registration of arbitrary integrity policies with the system (e.g., Biba Strict Integrity Policy) but no new policies are put forward.

The integrity control model for operating systems presented by [23] employs a roles based approach to integrity protection. The concept of a state machine and rule governed state transitions are coupled with two integrity axioms to model a system and ensure that its integrity is preserved from state to state. It should be noted however, that the listed axioms are essentially the same as those of the Biba Ring Policy (no write up) as are the elements of the model (users, subjects, objects, integrity levels etc.). Roles are merely a means for managing a user's responsibilities and are assigned integrity levels instead of the individual users/subjects. [22] proposed a model that enforces the Biba policy as the basic access control mechanism. But also the Clark-Wilson model is used to control and audit subjects' state transitions and manages run time adjustment of low-water-mark policy parameters. This hybrid approach claims to solve the issues of impracticality of the Biba model and the high complexity of Clark-Wilson, whilst retaining the merits of each.

[15] proposed a Least-Restrictive Enforcement of the CWSP based on graph representation, where their enforcement mechanism mediates read attempts only to prevent subject-violations, and write attempts only to prevent object violations. However, in their paper there is a strong mathematical confusion between the notion of the class, partition, equivalence binary relation and the transitivity property. Also, their graph representation is very complex for the implementation. The CWSP used in [16], is to fix inter-VM attack from competitors, which targets at the VMs running on the same physical machine, so each two competing VMs cannot be hosted in the same physical machines in that physical isolation. The authors use the conflict of interest and the graph colouring algorithm for the VM deployment. However, the authors were based on centralized control mechanism. 
Also, [17] used the CWSPM for the "Information Flow Control in Cloud Computing", at the IaaS level. Based on the concept of the conflict of interest which is partition (BN's model), however, it was a binary relation [8], and so they based on wrong model, and the same problem with two other work by [8] and [18] in Cloud Computing.

[7] based their idea on the access query type of the subject to the objects and the philosophy of the Chinese wall security policy CWSP. Its rule is the building of the walls between the competing companies. In their model, the authors have two types of walls placement, the first type consists of walls that are built around the subject, and the second around the object. They cannot find inside the same wall two data related to two competing objects.

\section{METHODOLOGY}

The method used in developing a multi-step permission based delegation model with Chinese wall security model is hereby discussed.

\subsection{Chinese Wall Simple Security Condition}

The Subjects are active entities accessing protected objects while the Objects are Data organized according to 3 levels: Information, Dataset and Conflict-of-Interest (CoI) classes. All object information is arranged hierarchically as shown in figure 1 . Let COI be a set conflict of interest classes whose element are also sets.

$\mathrm{COI}=\left\{\mathrm{CD}_{1}, \mathrm{CD}_{2}, \mathrm{CD}\right.$

where

$\mathrm{COI}(\mathrm{O})$ represent the $\mathrm{COI}$ class that contains object $\mathrm{O}$ and let $\mathrm{CD}(\mathrm{O})$ be the company dataset that contains object $\mathrm{O}$, then we have the following rules.

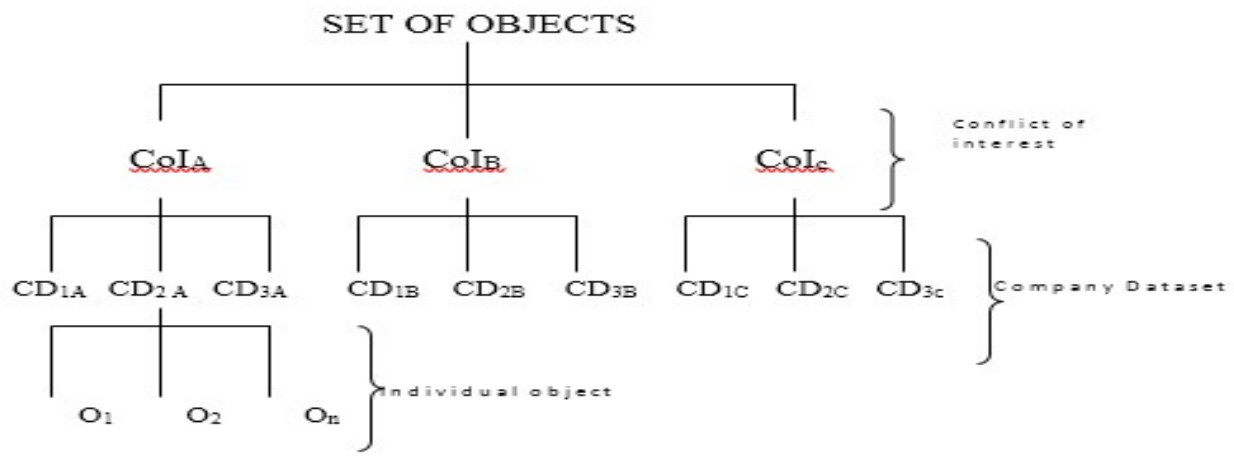

Figure 1: CWSP Object composition

(i) Read rule Policy

A subject or user $\mathrm{S}$ can read an object $\mathrm{O}$ if and only if any of the following holds:

- There is an object $\mathrm{O}^{\prime}$ such that $\mathrm{S}$ has accessed to $\mathrm{O}^{\prime}$ and $\mathrm{CD}\left(\mathrm{O}^{\prime}\right)=\mathrm{CD}(\mathrm{O})$.

- $\forall \mathrm{O}^{\prime} \in \mathrm{PR}(\mathrm{S}) \Rightarrow \mathrm{COI}\left(\mathrm{O}^{\prime}\right) \neq \mathrm{COI}(\mathrm{O})$. $\mathrm{O}$ is a sanitized object.

Where $\mathrm{PR}(\mathrm{s})$ is a set of an object $\mathrm{O}$ read by a subject or a user s 
A subject $\mathrm{S}$ can write an object $\mathrm{O}$ if:

- $\mathrm{S}$ can read $\mathrm{O}$ according to the Read Rule; and

- S can write to an object if all the (unsanitized) objects it can read are in the same dataset.

\subsection{Formal Definition of permission based delegation model}

Definition 1: U, RR, TDR, P, O, OBJ and T are set of users, regular role, temporary delegation role (created by delegator), permissions, operations, object, and time respectively.

- $\mathrm{P} \subseteq \mathrm{O} \times \mathrm{OBJ} ; \mathrm{P}$ is a subset of Cartesian sets of operation $\mathrm{O}$ and object $\mathrm{OBJ}$

- $\mathrm{R}=\mathrm{RR} \cup \mathrm{TDR} ; \mathrm{R}$ is a union set of regular roles and temporary delegation role.

- $\mathrm{PRA} \subseteq \mathrm{RR} \times \mathrm{P}$; PRA (permission regular-role assignment) is a subset of regular role and permissions.

- PDA $\subseteq$ TDR $\times$ P; PDA (permission delegation-role assignment) is a subset of a temporary delegation role and permission.

- PA = PRA U PDA; PA (permission-role assignment) is the set union of permission regular-role assignment and permission delegation-role assignment. Thus PRA $\cap$ PDA $=\emptyset$

- $\mathrm{URA} \subseteq \mathrm{U} \times \mathrm{RR}, \mathrm{URA}$ (user regular-role assignment) is a subset of users and regular role.

- $\mathrm{UDA}_{\mathrm{n}} \subseteq \mathrm{U} \times$ TDR UDA (user delegation-role assignment) is a subset of users and regular role. Where $\mathrm{n}$ is number of steps

Definition 2: Original members Users_O(r) are the members which originally assigned to the role by the system administrator. Hence, A mapping USER_O: $\mathrm{RR} \rightarrow 2^{\mathrm{U}}$ is a function mapping each regular role to set of users $U$ that is USER_O $(r)=\{u \mid$ where $r \in R R$ and $u \in U$ then $(u, r) \in U R A\}$.

Definition 3: Delegated members Users_D(r) are the members which are assigned to the role by other original members (that is assigned by delegation). Hence, A mapping USER_D : TDR $\rightarrow 2^{\mathrm{U}}$ is a function mapping a set of Temporary delegation role that was delegated to a user $\mathrm{u}$ at step $\mathrm{n}$ where $\mathrm{n}$ is number of steps.

Thus, USER_D $(\mathrm{tdr})=\left\{\mathrm{u} \mid\right.$ where $\mathrm{u} \in \mathrm{U}$ and $\operatorname{tdr} \in \mathbf{T D R}$ then $\left.(\mathrm{u}, \mathrm{tdr}) \in \mathrm{UDA}_{\mathrm{n}}\right\}$

Definition 4: In general USER: $\mathrm{R} \rightarrow 2^{\mathrm{U}}$ is a function mapping each role to set of users $\mathrm{U}$ Users $(\mathrm{r})=\{\mathrm{u} \mid$ where $r \in R$ and $u \in U$ then $(u, r) \in U A\}$.

Definition 5: A mapping Own: $\mathrm{U} \rightarrow 2^{\mathrm{TDR}}$ is a function mapping a user to a set of Temporary delegation role which he/she created. Own $(u)=\{t d r \mid$ where $u \in U$ and tdr $\epsilon$ TDR $\}$.

Definition 6: Two roles $r$ and r' are conflict with each other, if $(\exists \mathrm{p}, \mathrm{p} \in \mathcal{P})$ such that $(\mathrm{r}, \mathrm{p}) \in \mathrm{PA}$ and $\left(\mathrm{r}^{\prime}\right.$, p') $\in \mathrm{PA}$, such that $\mathrm{p} \leftrightarrow \mathrm{p}$ '. This is denoted as $\mathrm{r} \leftrightarrow \mathrm{r}$. A set of roles that conflict with $r$ can be denoted as $\Re$, where $\forall r_{i} \in \Re, r \leftrightarrow r_{i}$

Definition 7: For two permissions $\mathrm{p}_{1}=\left(\mathrm{o}_{1}, \mathrm{obj}_{1}\right)$ and $\mathrm{p}_{2}=\left(\mathrm{o}_{2}, \mathrm{obj}_{2}\right)$, if $C O I\left(\mathrm{obj}_{1}\right)=C O I\left(\mathrm{obj}_{2}\right)$ and $C D$ $\left(o b j_{1}\right) \neq C D\left(o b j_{2}\right)$, then $p_{1}$ and $p_{2}$ conflict with each other, which is denoted as $\mathrm{p}_{1} \leftrightarrow \mathrm{p}_{2}$

Hence, UA = URA U ...U UDA

$\mathrm{UA}_{\mathrm{ti}}$ is the $\mathrm{UA}$ at ti time point.

$\mathrm{UA}=\mathrm{U}_{\mathrm{t}}$

$\mathrm{UAH}=\mathrm{UA} \cup \ldots \cup \mathrm{UA}_{\star}$ : a relation of user-role assignment history. 


\subsubsection{Hierarchy Component of PBDM}

Figure 2 shows basic components of PBDM. In PBDM, roles are partitioned into regular roles (RR) and delegation roles (TDR). UA is also partitioned into user-regular role assignment (URA) and user delegation role assignment (UDA). PA is similarly separated into permission regular-role assignment (PRA) and permission delegation-role assignment (PDA).

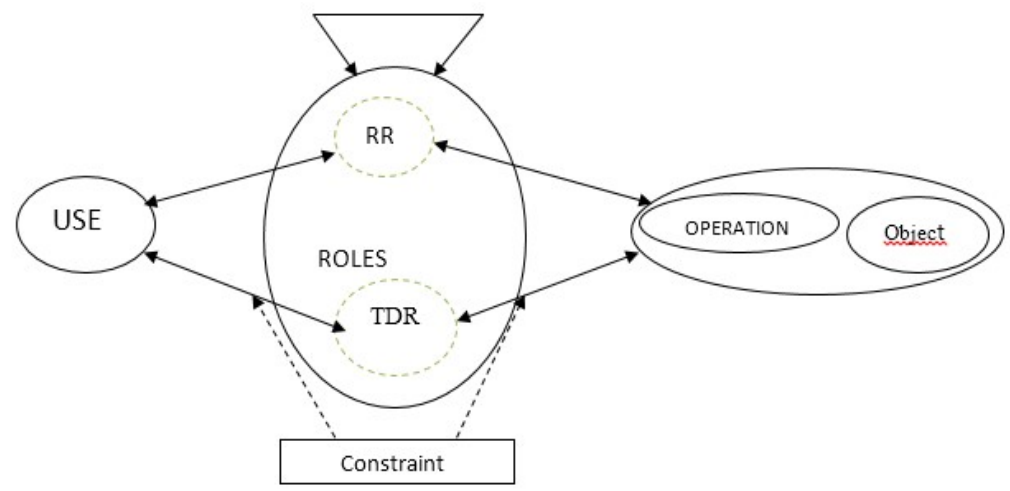

Figure 2: PBDM Hierarchy

(i) First step delegation

A delegation with strong CWSP can be denoted as a relation Can-delegate $\subseteq \mathbf{U} \times \mathbf{R} \times \mathbf{U}$ a successful delegation of role $r \in R R$ from delegator $u \in U$ to delegate $u^{\prime} \in U$ implies that (u, r, $\left.\mathrm{u}^{\prime}\right) \in$ can-delegate iff we have (u, r) $\in$ URA such that $\forall r_{i} \in \Re, \nexists \quad\left(u^{\prime}, r_{i}\right) \in U A H$

(ii) n-Number of steps delegation

Further delegation with strong CWSP can be denoted as a relation Can-further delegate $\subseteq \mathrm{U} \times \mathbf{R} \times \mathbf{U}$ a successful furthering of delegation $\operatorname{tdr} \in$ TDR from delegator $u \in U$ to delegate $u^{\prime} \in U$ implies that (u, tdr, $\left.u^{\prime}\right) \in$ Can-further delegate with the restriction of the number of steps defined by the original owner if we have $(\mathrm{u}, \mathrm{tdr}) \in \mathrm{UDA}_{\mathrm{n}}$ such that $\forall \mathrm{r}_{\mathrm{i}} \in \Re \mathrm{R}\left(\mathrm{u}, \mathrm{r}_{\mathrm{i}}\right) \in \mathrm{UAH}$.

\subsubsection{Revocation}

- Revocation perform by a delegator is denoted as a relation can-revoke $\subseteq \mathbf{U} \times \mathbf{R} \times \mathbf{U}$. (u,r,u') $\in$ canrevoke Mean a user u can revoke permission role from user u' successfully if $(\mathrm{u}, \mathrm{r}, \mathrm{u}$ ') $\in$ can-delegate or $\left(\mathrm{u}, \mathrm{r}, \mathrm{u}^{\prime}\right) \in \mathrm{c}$ can-further delegate.

- Revocation perform using time-out is denoted as a relation can-revoke $(\mathrm{t}) \subseteq \mathbf{U} \times \mathbf{R} \times \mathbf{U}$. (u,r,u') $\in$ canrevoke(t) Means permission role is revoked successfully if ( $\left.\mathrm{u}, \mathrm{r}, \mathrm{u}^{\prime}\right) \in$ can-delegate or $\left(\mathrm{u}, \mathrm{r}, \mathrm{u}^{\prime}\right) \in$ canfurther delegate and when duration as expired.

\subsubsection{Algorithm for Delegation and Revocation of Permission}

Algorithm 1 and 2 describes delegation procedure for first step and multi-step delegation. While Algorithm 3 and 4 gives revocation procedure of delegation by the delegator and using time out respectively. 


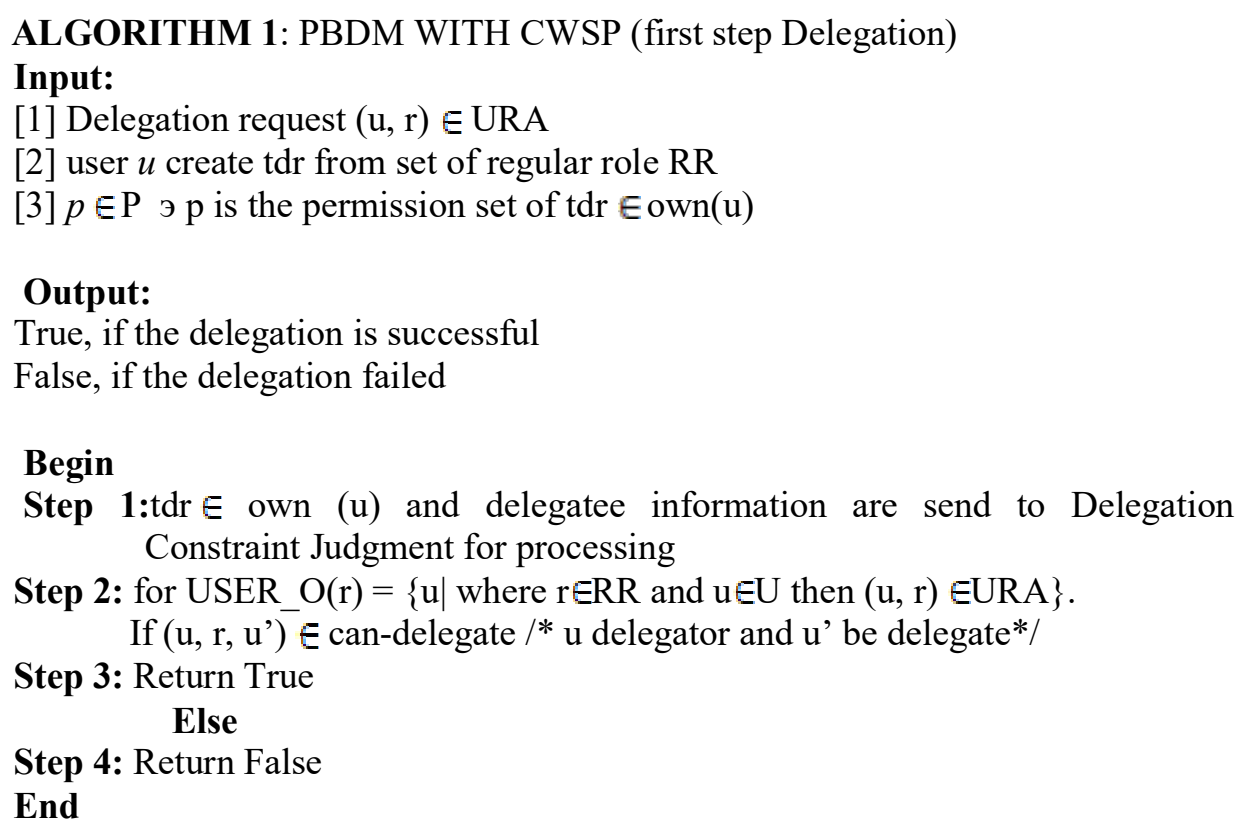

ALGORITHM 2: for Multi-step delegation

\section{Input:}

[1] a delegation request $(\mathrm{u}, \mathrm{tdr}) \in U \mathrm{UDA}$

[2] $u$ create tdr from set of regular role TDR

[3] $p \in \mathrm{P} \ni \mathrm{p}$ is the permission set of $\mathrm{tdr} \in \mathrm{own}(\mathrm{u})$

\section{Output:}

True, if delegation is successful

False, if delegation failed

\section{Begin}

Step 1: tdr $\in$ own (u) and delegatee information are send to Delegation Constraint Judgment for processing

Step 2: USER_D $(\mathrm{tdr})=\left\{\mathrm{u} \mid\right.$ where $t d r \in T D R$ and $u \in U$ then $\left.(\mathrm{u}, \mathrm{tdr}) \in \mathrm{UDA}_{\mathrm{n}}\right\}$.

If ( $\left.u, t \overline{d r}, u^{\prime}\right)$ Ecan-furtherdelegate/* u delegator and u' be delegate*/

Step 3: Return True

\section{Else}

Step 4: Return False

End 


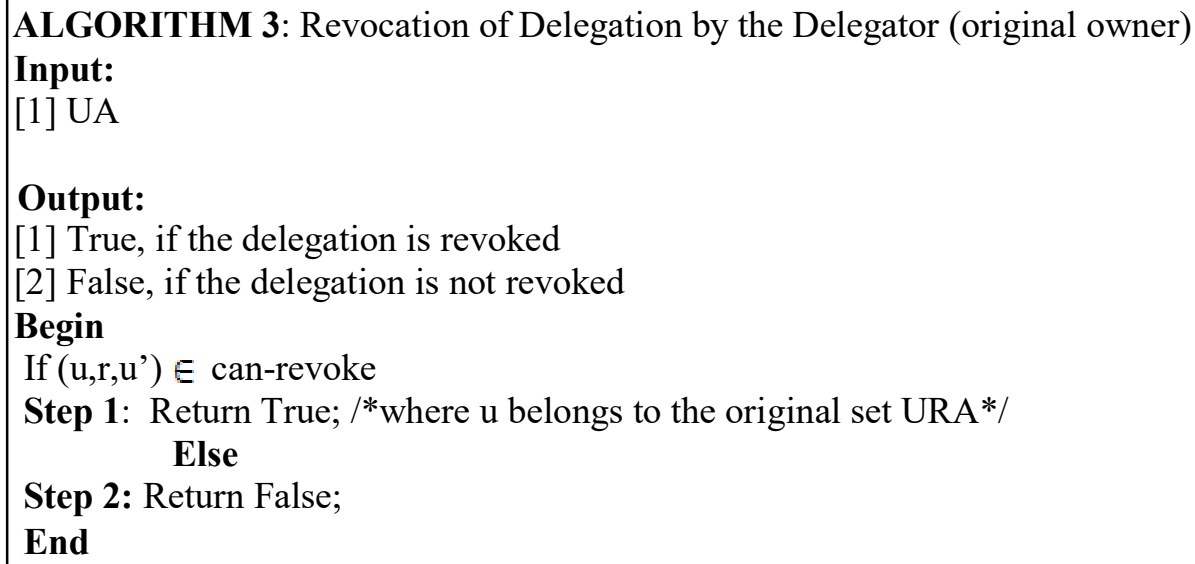

[1] True, if the delegation is revoked

[2] False, if the delegation is not revoked

Begin

If $\left(\mathrm{u}, \mathrm{r}, \mathrm{u}^{\prime}\right) \in$ can-revoke(t)

Step 1: Return True; $/ * \mathrm{t}$ is the duration after which the delegation is revoked*/

Else

Step2: Return False;

End

\subsubsection{Architectural and Web Design of PBDM with CWSP}

The figure 3 below shows how permission roles are delegated from the delegator user1 on system1 to delegatee according to the following 3 phases.

- User1 creates a temporary delegation role tdr $\in$ TDR and further assigns the read or write permission to the tdr he/she has created earlier.

- User1 chooses a user2 to assign the role permission

- Information of user2 is forwarded to the delegation constraint judgment to check if it satisfies the major constraint. (i.e. CWSP).

For further delegation of role permission from user2 to another user it requires moving through the same phase stated above. Figure 4 shows the web page Hierarchy. 
SYSTEM S (1

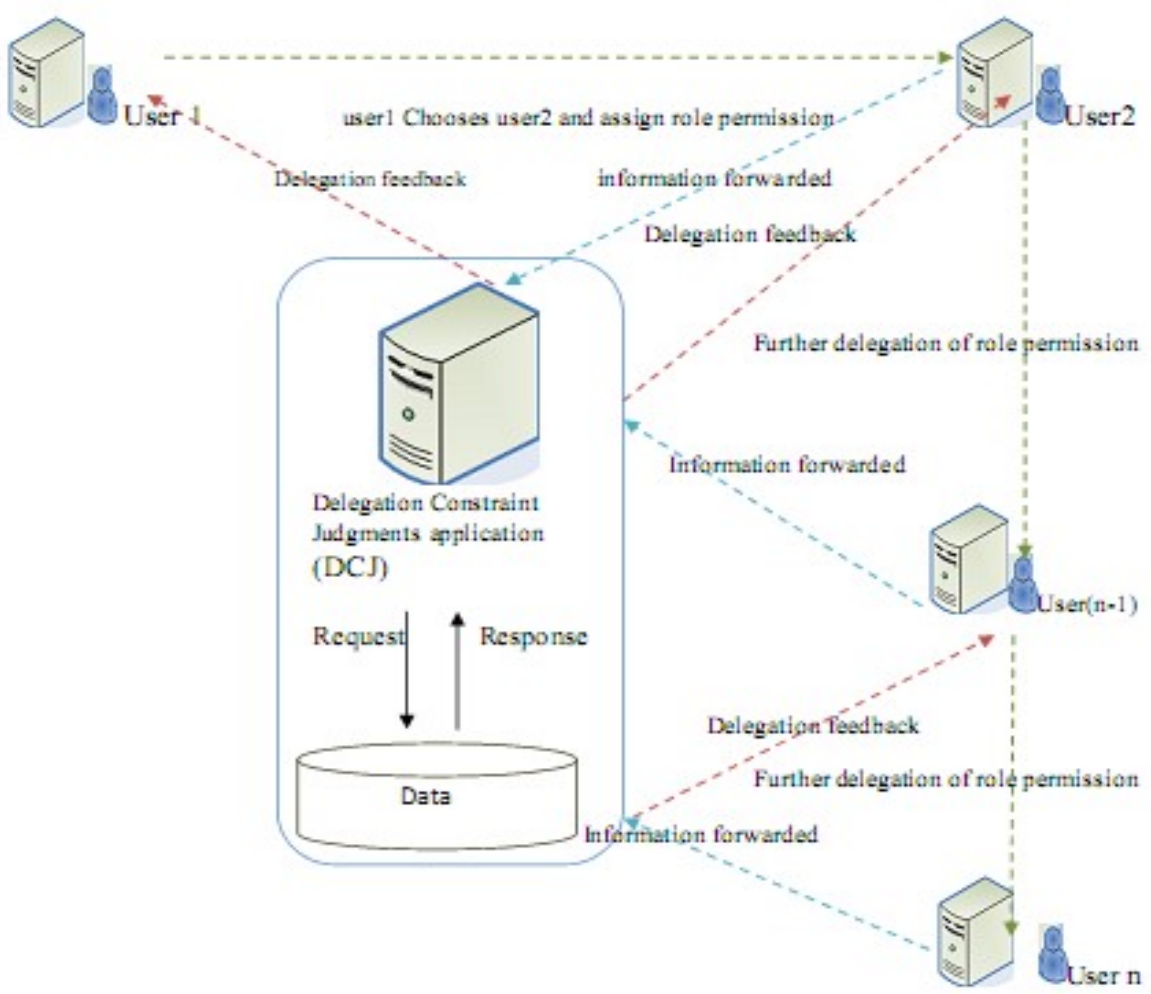

Figure 3: Architectural design of PBDM with CWSP

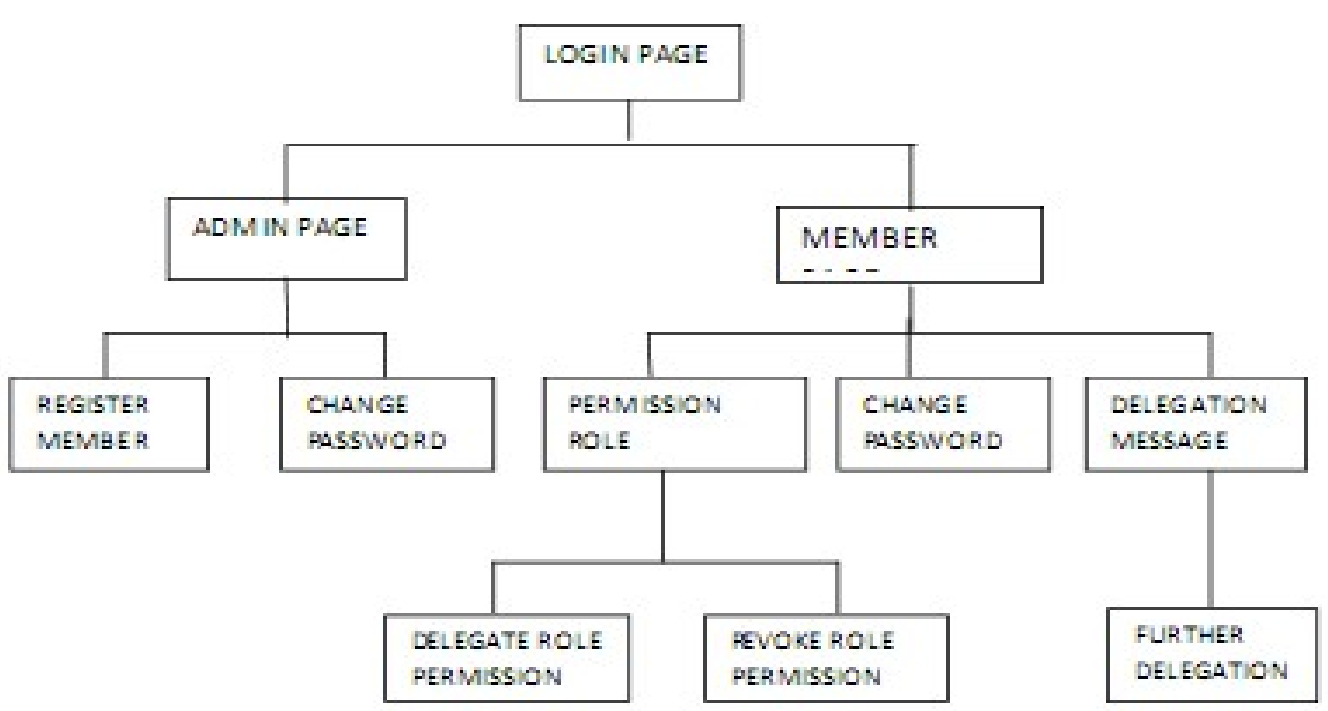

Figure 4: Web Design View 


\section{IMPLEMENTATION AND RESULT}

The design was implemented using PHP. Figure 5 displays the page of the companies where consultant is allowed to view any company's information at the first login. e.g. When "BACKLAYS BANK PLC" link is clicked the following page is display

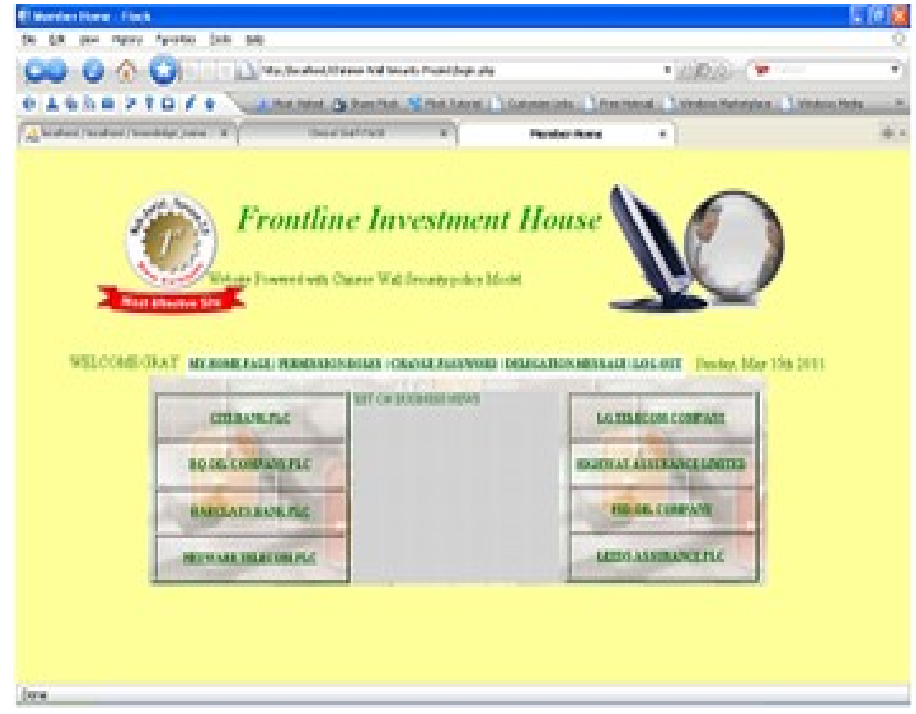

Figure 5: Company Information Page

While figure 6 shows the page where user (gray), delegating role permission "BARCLAYS BANK" to another user (Mustiey).

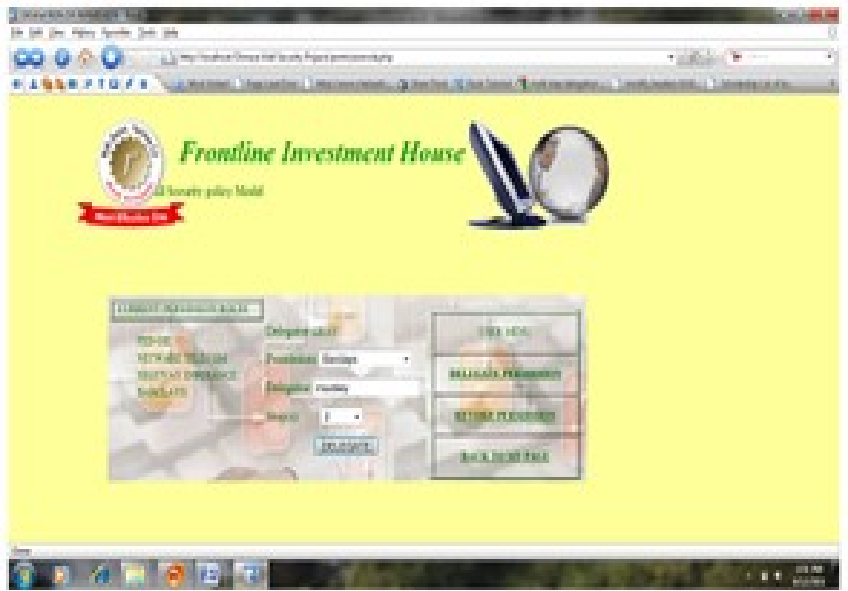

Figure 6: Delegation Page 


\section{CONCLUSION}

In this work, a commercial security policy was explored which represents the behaviour required of personnel who perform corporate analysis for financial institutions. It presents a Secured Multi-Step Permission Based Delegation Model with Chinese Wall Security Policy which explains how permission can be delegated in a multiple-step and considering CWSP as major constraint to be satisfied before delegation could be successful. Thus ensuring security of delegation with CWSP. The work further gave system architecture and implementation and finally a web application was developed to simulate PBDM model with CWSP.

In future, it might also be necessary to base the implementation on an aggressive Chinese wall security policy with permission based delegation model.

\section{References}

[1] Brewer David D.C. and Michael J. Nash, (1989), "The chinese wall security policy", In Proc. of the IEEE Symposium on Security and Privacy, Oakland, IEEE Press, 215228

[2] Byun, J., Sohn, Y. and Bertino, E. (2006) "Systematic control and management of data integrity", in SACMAT '06: Proceedings of the eleventh ACM symposium on Access control models and technologies, ACM Press, 101-110.

[3] Chunxiao Y, Fawen Y, Tinsen H, Haibo H, and Hong X, (2010), "Combining Permission Based Delegation Model with Chinese Wall"

[4] Crampton J. and Khambhammettu H. (2008). "Delegation in Role Base Access Control", international Journal of Information Security, 2008, 7(2), 123-136.

[5] David F. C. Brewer and Michael J. Nash. (1989). The ChineseWall Security Policy". In 1989 IEEE Symposium on Security and Privacy", pp. 206\{214. Oakland, CA, 1989.

[6] Fehis S. and Nouali O. (2015). A new Chinese Wall Security Policy Model Based On the Subject's Wall and Object's Wall, IEEE, 90-95

[7] Fehis S., Nouali O and Kechadi M. (2016). A New Distributed Chinese Wall Security Policy Model, Digital Forensics, Security and Law, 11(4), 149-168.

[8] Kesarwani, A., Gupta, C., Tripathi, M. M., Gupta, V., Gupta, R., \& Chaurasiya, V. K. (2011). Implementation of Chinese wall model in cloud computing for enhanced security. In Emerging trends in networks and computer communications (etncc), 2011 international conference, 411-413.

[9] Lin, T. Y. (1989). Chinese wall security policy- an aggressive model. In Computer security applications conference, 282-289.

[10]Lunt, T., Denning, D., Schell, R., Heckman, M. \& Shockley, W. (1990) “The sea-view security model”, IEEE Transactions on Software Engineering 16(6), 593-607

[11]Quan, P., Jason, J., Adrian, M. and Ed, D. (2008). "Commitment issues in delegation process", Australasian Information Security Conference (AISC2008), Wollongong, Australia, January 2008. Conferences in Research and Practice in Information Technology (CRPIT), 81.

[12] Samarati and Vimercati2 (2001). Access Control: Policies, Models, and Mechanisms, LNCS 2171, 137-196

[13]Sandhu R. S (1992). Lattice-Based Enforcement of Chinese Walls. Computers \& Security, 11 (1992), 753-763

[14] Schell, R. \& Denning, D. (1986) "Integrity in trusted database systems", in 9th National Computer Security Conference

[15] Sharifi A. and Tripunitara M. V. (2013). Least-restrictive enforcement of the Chinese wall security policy. SACMAT: $61-72$

[16]Tsai, T.-H., Chen, Y.-C., Huang, H.-C., Huang, P.-M., \& Chou, K.-S. (2011). A practical Chinese wall security model in cloud computing. In Network operations and management symposium (apnoms), $201113^{\text {th }}$ Asia-pacific, 1(4). 
[17]Wu, R., Ahn, G.-J., Hu, H., \& Singhal, M. (2010). Information flow control in cloud computing. In Collaborative computing: Networking, applications and work sharing (collaboratecom), 2010 6th international conference, 1\{7).

[18]Xie, X., Ray, I., Adaikkalavan, R., \& Gamble, R. (2013). Information flow control for stream processing in clouds. In Proceedings of the 18th ACM symposium on access control models and technologies, 89100 .

[19] Ye C., Hu H. and Xiang H. (2011). Towards Secure Delegation with Chinese Wall Security Policy (CWSP), Journal of Networks, 6 (8).

[20] Ye C., Wu W and Fu Y (2006). "An Attribute-Based Extended Delegation Model”, Journal of Computer Research and Development,2 006,43(6), 1050-1057.

[21] Zhang X, Oh . and Sanhhu R, (2003) "PBDM: A Flexible Delegation Model in RBAC", In Proc. of the SACMAT'03, Como, Italy: ACM press, 149 - 157.

[22] Zhou-Yi, Z., Ye-Ping, H. \& Hong-Liang, L. (2010) "Hybrid mandatory integrity model composed of biba and clark-wilson policy”, Ruan Jian Xue Bao (Journal of Software) 21(1), 98-106.

[23] Zun, L., Tao, W. and Wei-hua, L. (2009) "An integrity control model for operating system", in Management and Service Science, 2009 MASS ’09 International Conference on, 1-4. 